\title{
Near Exit Plane Effects Caused by Primary and Primary-Plus-Secondary Tabs
}

\author{
D. G. Bohl* and J. F. Foss ${ }^{\dagger}$ \\ Michigan State University, East Lansing, Michigan 48824
}

\begin{abstract}
Triangular-shaped tabs, attached to the separation lip of a nozzle, have been shown to provide substantial increases in the convective transport-mixing in a jet's near field. Detailed information is reported on the basic features of such tab flows made available by 1) the large-scale tab and 2) the finely resolved pressure/velocity measurements. Secondary tabs were placed next to, and pointed away from, the primary tab; the motivation was to enhance the outward directed flow from the jet's core region. The strong mixing enhancement, provided by these optimally oriented (pitch angle $=40 \mathrm{deg}$ ) secondary tabs, is detailed.
\end{abstract}

\section{Introduction}

$\mathbf{P}$ ASSIVE mixing tabs, placed at the exit plane of a jet, have been shown to 1) eliminate the phenomenon of supersonic screech and 2) enhance the lateral convective transport (mixing) of ambient and jet-core fluid. Bradbury and Khadem ${ }^{1}$ are credited with the first demonstration of phenomenon 2 , and they correctly surmised that the modifications to the jet's near field could reduce the production of jet noise. Subsequent investigations, e.g., Refs. 2 and 3, have reinforced and clarified the mechanisms for these beneficial effects. The present study is only concerned with the second phenomenon, and it is restricted to the incompressible regime. Significant original contributions as well as a comprehensive literature review for this second attribute of tabbed jets are provided by Reeder and Samimy. The present authors will not attempt to add to the summary of the prior work as given in Ref. 4; the specific attributes of tabbed jets that are relevant to the present results are noted subsequently.

Figure 1 provides a schematic representation of the time-average streamwise vorticity $\overline{\omega_{x}}$ that exists at the exit plane of a tabbed jet. The circulation pattern, which results from the $\overline{\omega_{x}}$ distributions, is shown. The indicated tab is referred to as a delta tab by Zaman et al. ${ }^{3}$ The counter-rotatingmotions at the sides of the tab can be explicitly traced to the coupled upstream effects: 1) the reorientation of the upstream laminar boundary-layervorticity, $\overline{\omega_{y}}$, as described by the term $\overline{\omega_{y}} \partial \bar{u} / \partial y$ in the transport equation for $\overline{\omega_{x}}$, viz,

$$
\begin{aligned}
& \bar{u} \frac{\partial \overline{\omega_{x}}}{\partial x}+\bar{v} \frac{\partial \overline{\omega_{x}}}{\partial y}+\bar{w} \frac{\partial \overline{\omega_{x}}}{\partial z}=v \nabla^{2} \overline{\omega_{x}}+\overline{\omega_{x}} \frac{\partial \bar{u}}{\partial x}+\overline{\omega_{y}} \frac{\partial \bar{u}}{\partial y} \\
& +\overline{\omega_{z}} \frac{\partial \bar{u}}{\partial z}+\frac{\partial}{\partial x}\left(\frac{\partial \overline{u^{\prime} v^{\prime}}}{\partial z}-\frac{\partial \overline{u^{\prime} w^{\prime}}}{\partial y}\right)+\frac{\partial^{2}}{\partial y \partial z}\left(\overline{v^{\prime 2}}-\overline{w^{\prime 2}}\right) \\
& +\left(\frac{\partial^{2}}{\partial z^{2}}-\frac{\partial^{2}}{\partial y^{2}}\right) \overline{v^{\prime} w^{\prime}}
\end{aligned}
$$

and 2) vorticity that has been introduced into the flow by the action of the pressure gradient established upstream of the tab.

The basis for item 2 has been describedin detail by Lighthill ${ }^{5}$ and Panton. ${ }^{6}$ The presentation by Panton is considered to be particularly instructivegiven its emphasis on the difference between the presence of vorticity at the wall (analogous to wall temperature) and the flux

Presented as Paper 96-0545 at the AIAA 34th Aerospace Sciences Meeting, Reno, NV, Jan. 15-18, 1996; received May 29, 1997; revision received Sept. 16, 1998; accepted for publication Oct. 1, 1998. Copyright (C) 1998 by the American Institute of Aeronautics and Astronautics, Inc. All rights reserved.

* Graduate Student, Department of Mechanical Engineering. Student Member AIAA.

${ }^{\dagger}$ Professor, Department of Mechanical Engineering. Member AIAA. of vorticity from the surface into the surrounding fluid (analogous to heat flux). Using the nomenclature ( $\sigma_{i}$ as the flux and $n_{j}$ as the wall normal unit vector) from that reference, Panton writes [Ref. 6 , Eq. (13.7.6)]

$$
\sigma_{i}=-n_{j} \frac{\partial \omega_{i}}{\partial x_{j}}
$$

and using the momentum equation at the wall, he writes [Ref. 6, Eq. (13.7.8)]

$$
\frac{\partial p}{\partial x_{i}}=-\mu \varepsilon_{i j k} \frac{\partial \omega_{k}}{\partial x_{j}}
$$

For the present flowfield (Fig. 1), and using the understanding that Eq. ( $2 \mathrm{~b}$ ) is equivalent to stating that the vorticity enters the flow field as if it rolls down the pressure hill, $\overline{\omega_{y}}>0$ results from the positive $\partial p / \partial x$ values. Similarly, for the $y<0$ region that is shown in the figures here, $\overline{\omega_{x}}<0$ results from the positive $\partial p / \partial y$ values of the pressure hill. It is parenthetically noted that the understanding of the source of the motions marked $\mathrm{II}_{-}$and $\mathrm{II}_{+}$in Fig. 1 is contrary to that suggested by Reeder and Samimy, ${ }^{4}$ who have suggested that the transport equation for vorticity, i.e., Eq. (1), can account for the pattern shown in Fig. 1. It is explicitly noted that the mechanism 2 must be invoked to characterize the streamwise vorticity (characterized by motions II) that is present at $x=0$ for the tabbed jet flow. Specifically, the (baratropic form of the) vorticity transport equation, whether time averaged as in Eq. (1) or in the instantaneous form,

$$
\frac{\mathrm{D} \boldsymbol{\omega}}{\mathrm{D} t}=\boldsymbol{\omega} \cdot \nabla \boldsymbol{V}+v \nabla^{2} \boldsymbol{\omega}
$$

describes the rate of change of vorticity A within the flowfield. It does not constitute a source of new vorticity, albeit term B can be considered to be a source term for a component, e.g., $\omega_{x}$, given a preexisting component, e.g., $\omega_{y}$.

Previous work by the present authors, ${ }^{7}$ and presented here as the results from a single, i.e., a primary, tab, led to the recognition that secondary tabs, as shown in Fig. 1, could be used to further enhance the mixing induced by the primary tab. This innovation, which was reported in Ref. 8, has stimulated work by Yu and Hou, ${ }^{9}$ who investigated four sets of tabs in a square jet. The complexities of their ${ }^{9}$ four-tab geometry preclude useful direct comparisons with the present study.

The basic motivation for the addition of the secondary tabs came from the observation of the induced outflow at the center of the concentrated streamwise vortex motions $\mathrm{I}_{-}$and $\mathrm{II}_{-}$and $\mathrm{I}_{+}$and $\mathrm{II}_{+}$ of Fig. 1. It was reasoned that a physical surface, placed next to the primary tab and directed toward the ambient region, would enhance this outward displacement of core fluid and, correspondingly, induce a greater inflow from the ambient region near $y=0$. Surface streaking observations, provided by Bohl and Foss ${ }^{10}$ but not 


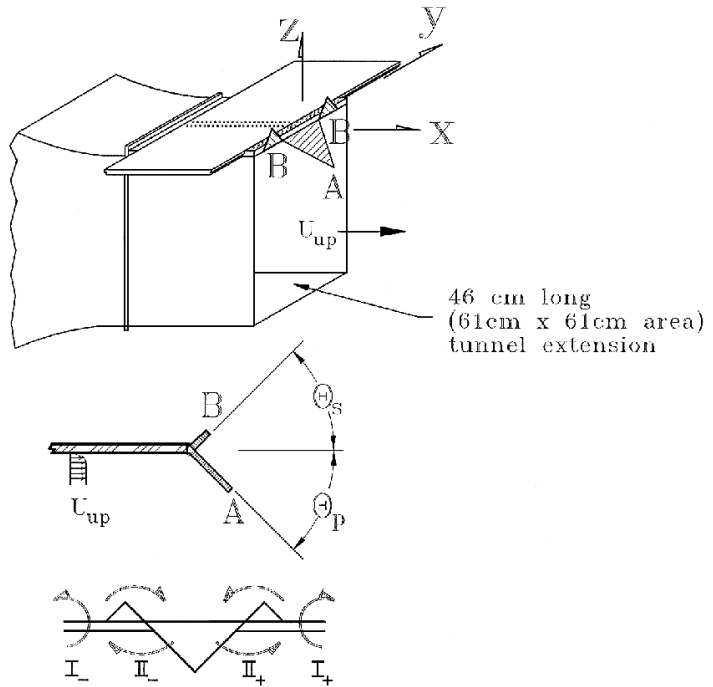

Fig. 1 Schematic representation of the $P \& S$ tabbed jet: primary A plus secondary B tabs.

reported here, show that the jet fluid attaches to the secondary tab for the optimum performance condition. This attachment helps to explain the efficacy of the secondary tabs.

The experimental equipment and procedures are identified in the second section, and the results that document the basic jet (no tab), the flowfield of the primary tab, and the primary plus secondary $(\mathrm{P} \& \mathrm{~S})$ tabbed geometry are presented in the third section. The secondary tabs were arbitrarily selected to be of the same geometry (an equilateral triangle) as that of the primary tab. Also, the base of the secondary tab, $b_{s}$, was selected to be $0.5 b$. The incidence angle $\theta_{s}$ was, however, selected on the basis of direct measurements as discussed in the third section.

\section{Experimental Equipment and Procedures}

A closed-circuit Engineering Laboratory Design wind tunnel was modified by the removal of the $61 \times 61 \times 244 \mathrm{~cm}^{3}(2 \times 2 \times$ $8 \mathrm{ft}^{3}$ ) test section, which created a Goettingen-style wind tunnel. A 46-cm-long tunnel extension, shown in Fig. 1, was added to the exit of the modified tunnel. This created a square freejet that used the tunnel flow as the core fluid and the laboratory as the source of ambient, i.e., entertainment, fluid. The tunnel extension was machined to allow the primary tab to be mounted in the exit plane of the jet. The tab was oriented at $\theta_{p}=45 \mathrm{deg}$ into the high-speed core flow, see Fig. 1. (Zaman et al. ${ }^{3}$ determined that $45 \mathrm{deg}$ provided the conditions for maximum mixing of the primary-only configuration. This result was not examined further with the addition of secondary tabs.) The two secondary tabs could be positioned at arbitrary angles with respect to the $x$ axis. Additionally, the top surface of the extension was fitted with two rows of static pressure taps $(-1.9 \leq x / b \leq-0.15)$, as shown in Fig. 1. This portion of the top surface was positionable in the $y$ direction to permit the static pressure distribution $P(x, y)$ to be measured. Note that the two additional rows of taps, $x / b=-0.025$ and -0.125 , which extended over a span of $-1.4 \leq y / b \leq 1.4$, were fixed, i.e., they were not part of the movable surface, as shown in Fig. 1. The downstream entrance to the modified tunnel was fitted with half-round flow conditioners.

The tab, machined from 3-mm- ( $\frac{1}{8}$-in.-) thick aluminum stock, had a base length $b$ of $200 \mathrm{~mm}$. The tab was placed in the flow at the center point of the top surface of the tunnel. The geometric blockage of the jet exit plane, i.e., the projected area of the tab in the tunnel exit plane, was $6.6 \%$ for the present tab geometry. All experiments were performed with a nominal approach velocity $U_{\text {up }}=10.5 \mathrm{~m} / \mathrm{s}$, which provided a Reynolds number based on the tab base length of $1.4 \times 10^{5}$. ( $U_{\text {up }}$ is the freestream velocity upstream of the tab.)

Hot-wire data were taken using DISA 55M10 and TSI 1750 anemometers. The noise levels of these anemometers were found to be \pm 2.0 and $\pm 1.5 \mathrm{mV}$ peak to peak, respectively. The equivalent noise levels were less than $0.3 \%$ of $U_{\text {up }}$. All hot-wire probes used in this study were fabricated (in-house) at the Michigan State University Turbulent Shear Flows Laboratory. Hot-wire sensors were fabricated from $5-\mu \mathrm{m}$-diam tungsten wires. The tungsten wire spanned a length of $3 \mathrm{~mm}$ with 1 -mm active length centered between two 1-mm-long regions of nominally 50- $\mu$ m-diam copper-plated sections. Prong lengths of nominally $20 \mathrm{~mm}$, forward of the probe bodies, supported these composite wires.

Pressure data were taken using a 1-torr MKS Baratron pressure transducer. Data acquisition was accomplished via a 486-66 personal computer clone with an Analogic Fast-16 A/D board. An eight-channel sample and hold card was used in conjunction with the $\mathrm{A} / \mathrm{D}$ card to provide true simultaneous sampling. The A/D resolution was 16 bit with an inherent noise level of \pm 1 bit $(0.31 \mathrm{mV}$ on a $\pm 10-\mathrm{V}$ scale).

\section{Experimental Methods}

\section{Hot-Wire Probe Considerations}

Data from the single sensorhot-wire probes were processedusing the modified Collis and Williams ${ }^{11}$ relationship

$$
E^{2}=A+B Q^{n}
$$

This form is also used for an $x$ array of sensors where $A, B$, and $n$ are functions of the pitch angle $\gamma$. The minimum calibration speed $(1.5 \mathrm{~m} / \mathrm{s})$ was selected on the basis of the linearity specification ( $0.1 \%$ of full scale) for the 1-torr pressure transducer. Specifically, the measured pressure difference at $1.5 \mathrm{~m} / \mathrm{s}$ is 10 times this uncertainty value.

The data from the $x$-array hot-wire probes were processed using the speed wire/angle wire scheme described by Foss et al. ${ }^{12}$ The probe was calibrated using 6 flow speeds $(1.5-13 \mathrm{~m} / \mathrm{s})$ and 13 angles $(-36 \leq \gamma \leq 36 \mathrm{deg}$ in 6-deg increments). In this study two $x$ arrays were used simultaneouslyto provide $(u, v)$ and $(u, w)$ at each sample time and at locations that were separated by nominally $2.12 \mathrm{~mm}$ $(\approx 1 \%$ of the tab width $b)$. The two values of $u$ from the $x$ arrays were averaged to provide $u$ for the probe.

A single-sensorhot-wire probe was used to measure the flow velocity for the untabbed jet at $x / b=0.7,1.2$, and 2.0 and at $x / b=0.7$ for the tabbed jet because the pitch angles in the flow were too extreme for $x$ array measurements at this location. The single-wire data contain contributions from both $u$ and $w$. The $x$ arrays were used to measure $[u(t), v(t)]$ and $[u(t), w(t)]$ at $x / b=1.2$ and 2.0 (Ref. 10) albeit only data from $x / b=1.2$ are presented.

\section{Streamwise Vorticity Calculation}

The temporally averaged streamwise vorticity is defined as

$$
\overline{\omega_{x}}=\frac{\partial \bar{w}}{\partial y}-\frac{\partial \bar{v}}{\partial z}
$$

The derivatives in Eq. (3) were evaluated using the averaged data from the $x$ arrays via the second-orderfinite differencemethod. Note that the distances between the mesh points of the measurement grid were $\Delta y=\Delta z=0.075 b$.

\section{Cross-Vane Vorticity Probe}

A cross-vane vorticity probe, shown in Fig. 2, was used to provide information about the streamwise vorticity field in regions of the flowfield where the pitch/yaw angles were sufficiently large that $x$-array probes could not be used. The cross-vane probe was placed

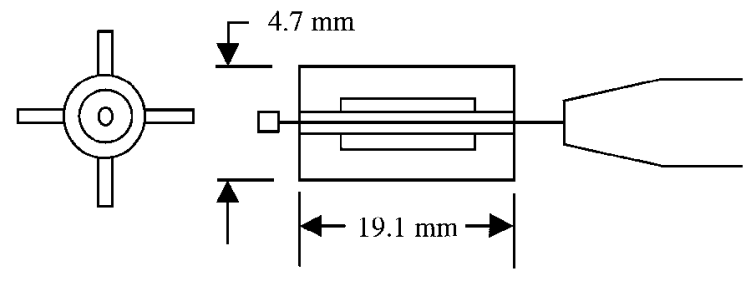

Fig. 2 Cross-vane streamwise vorticity probe. 
into the flow with the probe axis oriented in the streamwise direction. The blades of the probe rotated in the presence of nonzero streamwise vorticity $\omega_{x}$ with sufficient magnitude to overcome the bearing friction of the device. This probe was used in a qualitative manner. The probe's spin direction, as well as its rate, i.e., fast or slow, were noted. These observations were used to determine the nominal boundaries of the regions of streamwise vorticity in the domain $0.1 \leq x / b \leq 0.4$. Reliable cross-vane observations could not be made at larger $x$ values as a result of the reduced strength of the vortex motions.

\section{Uncertainty Considerations}

The uncertainty in the hot-wire measurements was estimated using the calibration data as described in the following manner. The hot-wire probes were calibrated both before and after the data were acquired. The two sets of calibration data pairs, $E_{\mathrm{hw}}$ and $Q$, were combined, and the combined calibration data were used to provide the calibration coefficients $(A, B, n)$ in Eq. (4) for each wire and for each pitch angle $\gamma$. The resulting standard deviation of the calibration data from the curve described by Eq. (4) represents the minimum uncertainty for the velocity component measurements. Note that this measure includes probe drift. If the standard deviations, as described earlier, for a single sensor were less than $0.05 \mathrm{~m} / \mathrm{s}$, the data for that run were accepted. If not, the run was repeated. Standard deviations of the $x$ array were limited to $\leq 0.15 \mathrm{~m} / \mathrm{s}$. These uncertainties in the velocity magnitudes represent 0.5 and $1.5 \%$ of the approach velocity $U_{\text {up }}$, respectively. The uncertainties in the computed angles were estimated by substituting the calibration voltages into the data processing routine. Typical error values of $0.1 \mathrm{deg}$ were obtained using this procedure. Reference 10 includes an enhanced discussion of the uncertainty considerations, as well as a complete record of the experimental data.

A second contribution to the uncertainty is related to the accumulation of an adequate number of independent samples. The present measurements were acquired for an elapsed time $T$, where $T$ can be best characterized as

$$
\left[(T)\left(U_{\text {up }}\right) / b\right]=1575
$$

If an independent sample were recovered following a delay of one convected body dimension $b$, and if the samples showed a Gaussian distribution, then a convergence to within $\sigma / \sqrt{ } N$ or $0.025 \sigma$ of the true mean value would be experienced for the sample size of $(N=1575)$. (Note that $\sigma$ is the standard deviation of the measured distribution, which is assumed to be nominally equal to the standard deviation of the actual distribution for this analysis.)

There are regions in the present flowfield where the flow velocities are below the minimum calibration speed $(1.5 \mathrm{~m} / \mathrm{s})$ of the probes. Measurements of the velocities below the calibration range require extrapolation of the calibration data. These measurements exhibit a greater uncertainty than do measurements that lie within the calibration range. The contour plots of the streamwise velocities are presented with a minimum contour of $0.15 U_{\text {up }}$ or a dimensional value of nominally $1.6 \mathrm{~m} / \mathrm{s}$, which is within the calibration range.

\section{Results and Discussion}

\section{Untabbed Geometry}

Approach Boundary-Layer Results

A survey of the boundary layer at the exit of the tunnel extension was conducted without the tab present (termed the untabbed geometry). The results are shown in Fig. 3. These data indicate an exit boundary layer thickness of $\delta=3 \mathrm{~mm}(0.015 b)$. The displacement thickness and momentum thickness are $\delta_{d}=1.27 \mathrm{~mm}(0.0064 b)$ and $\theta=0.52 \mathrm{~mm}(0.0026 b)$, respectively. These results show that the exit boundary layer is thin and laminar: $H=\delta_{d} / \theta=2.44$. There is a local maximum in the velocity profile at $y / \theta=7.5$ which is attributed to an inviscid acceleration near the bounding wall of the upstream tunnel contraction. The velocity profile suggests that $u / U_{\text {up }}$ approaches 1 for $y / \theta>10$; these boundary-layer data did not extend sufficiently far into the approach stream to observe $u(y)=U_{\text {up }}$. The Blasius profile is also shown in Fig. 3. Nominal

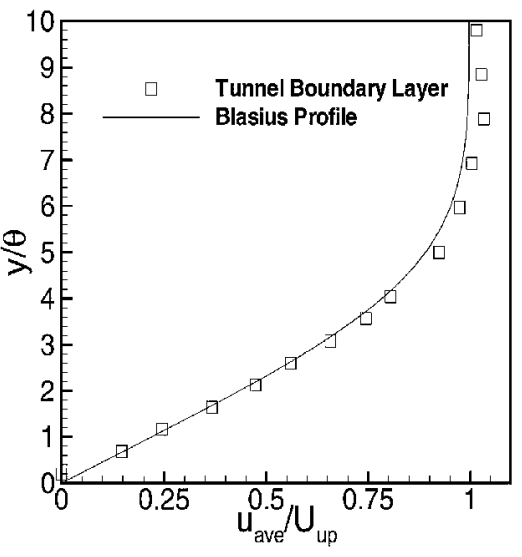

Fig. 3 Boundary-layer velocity profile at $x / b=-0.05$; note: $U_{\text {up }}=$ $10.5 \mathrm{~m} / \mathrm{s}$ (nominal for these data) and $\theta=3 \mathrm{~mm}$.

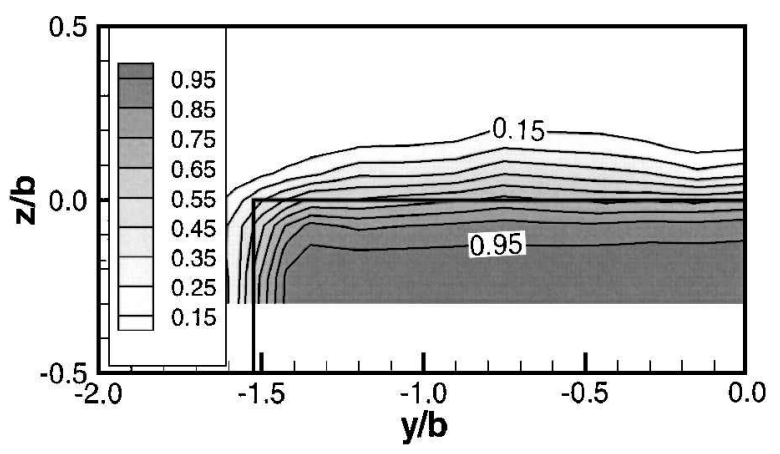

Fig. 4 Untabbed isotachs $(x / b=1.2)$.

agreement between the measured data and this reference case is observed.

\section{Streamwise Velocity Results}

Representative streamwise velocity results $(x / b=1.2)$ for the untabbed geometry are shown in Fig. 4. The shear layer, as described by the isotach lines, is aligned with the tunnel top and side walls at all three $(x / b=0.7,1.2$, and 2.0) measurement locations as shown in Ref. 10. As expected, the shear layer grows in size with increased downstream location, and it displays a greater penetration into the low-speed side (the ambient region) than into the high-speed side (the core region). The turbulence intensities (not included here; see Ref. 10) show a peak value of $\tilde{u}=0.18 U_{\text {up }}$ at all three measurement planes. These values are consistent with the turbulence intensity values in a single-streamshear layer (see, for example, Refs. 13-15).

\section{Tabbed Geometry}

\section{Upstream Results}

The upstream static pressure results for the P\&S tabbed jet are shown in Fig. 5. The pressure coefficient

$$
C_{p}=\left[P(x, y)-P_{\text {atm }}\right] / \frac{1}{2} \rho U_{\text {up }}^{2}
$$

is used to characterize these values. As described in the Introduction, a useful construct is to consider the vorticity flux into a flow at a physical surface to be described as if it is entering by rolling down a pressure hill. The entering vorticity is, therefore, tangent to the isobars. Its orientation at $x=0$ will, however, be governed by the transport equation (1).

The comparison of the primary and $\mathrm{P} \& \mathrm{~S}$ geometries is most clearly shown as presented in Fig. 6, which presents the difference contours. Specifically, Fig. 6 presents contours of $\Delta C_{p}$ where

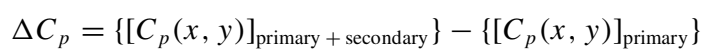

The principle difference between the two cases is the pressure minimum forward of the secondary tab. The mechanistic reasons for the 


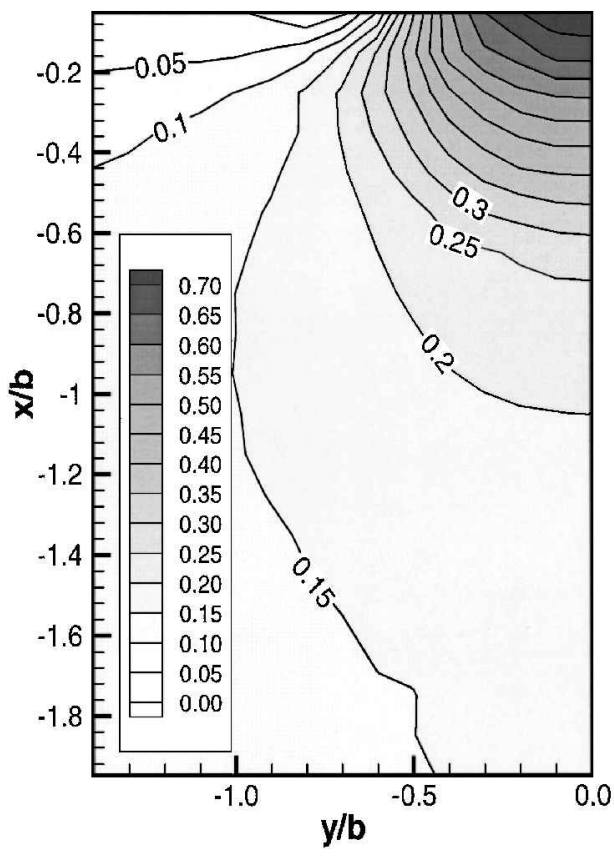

Fig. 5 Pressure coefficient $C_{p}$ values for the P\&S geometry.

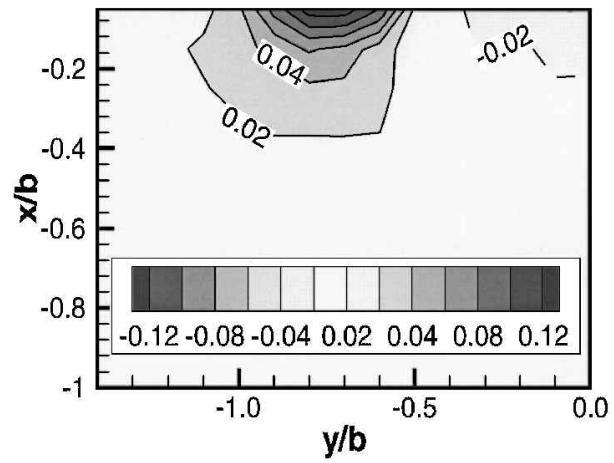

Fig. 6 Difference contours: $\left[C_{p}(\right.$ primary $\left.)-C_{p}(\mathrm{P} \& \mathrm{~S})\right]$ in the approach flow region.

modification can be readily appreciated in the context of the Euler $n$ equation

$$
\frac{\partial p}{\partial n}=-\rho \frac{V^{2}}{R}
$$

The relevant curvature, in this case, is that of the streamlines that approach the jet exit and follow the upward extension of this surface as defined by the secondary tab. This streamline pattern is qualitatively similar to that which is present in the case of the primary-only tab. (That is, it can be related to the side-by-side presence of the $\omega_{x}>0$, from the reoriented boundary layer, and the $\omega_{x}<0$, from the pressure hill, motions.) However, the attachment surface provided by the secondary tab strengthens this effect with the consequence of the pressure minimum as shown at $y / b=-0.8$.

The measured peak value (at $y=0$ ) of $C_{p}$ was 0.70 for the primary tab geometry and 0.72 for the $P \& S$ tab geometry. The peak values are located at the centerline of the tunnel $(y=0)$ and at the tap closest to the tunnel/tab junction $(x=0.05 b)$. It is inferred that the peak pressures, i.e., at locations closer to the tab, are slightly larger than the indicated values for the two cases. This inference derives from the positive slope of the $C_{p}(x, y=0)$ distributionat the farthest downstream measurement location $(x=-0.05 b)$.

The measured pressure values have also been processed to provide the magnitude of the pressure gradient on the bounding surface $(x<0, y, z=0)$. Specifically, the quantity $\mathrm{d} p^{*} / \mathrm{d} s^{*}$, which is defined as

$$
\frac{\mathrm{d} p^{*}}{\mathrm{~d} s^{*}}=\left[\left(\frac{\partial C_{p}}{\partial x / b}\right)^{2}+\left(\frac{\partial C_{p}}{\partial y / b}\right)^{2}\right]^{\frac{1}{2}}
$$

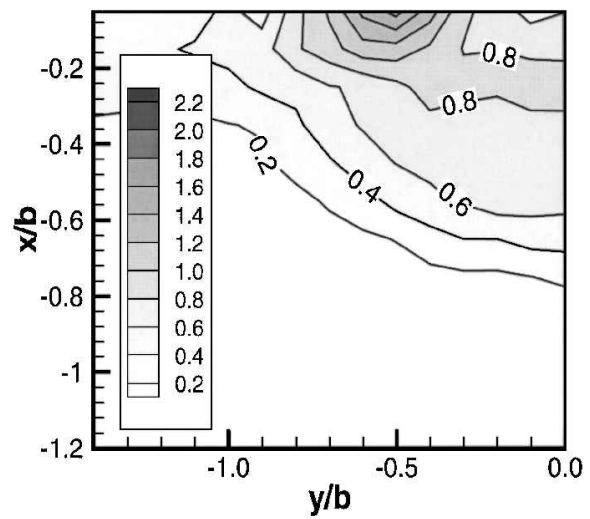

a) Primary tab

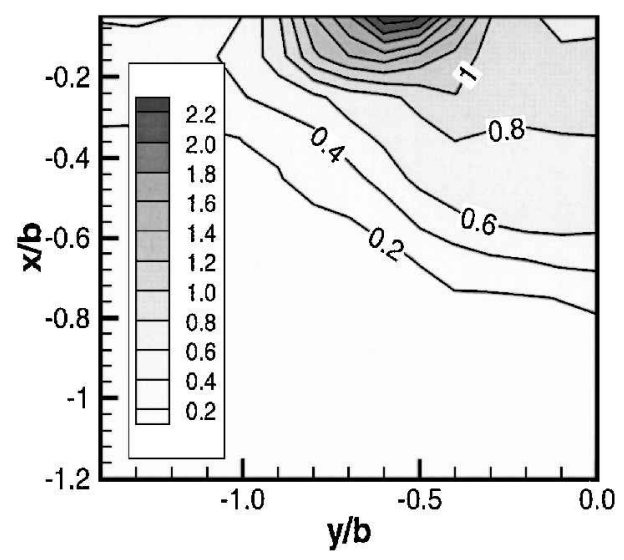

b) $P \& S$ configuration

Fig. 7 Surface pressure gradients; source terms for vorticity.

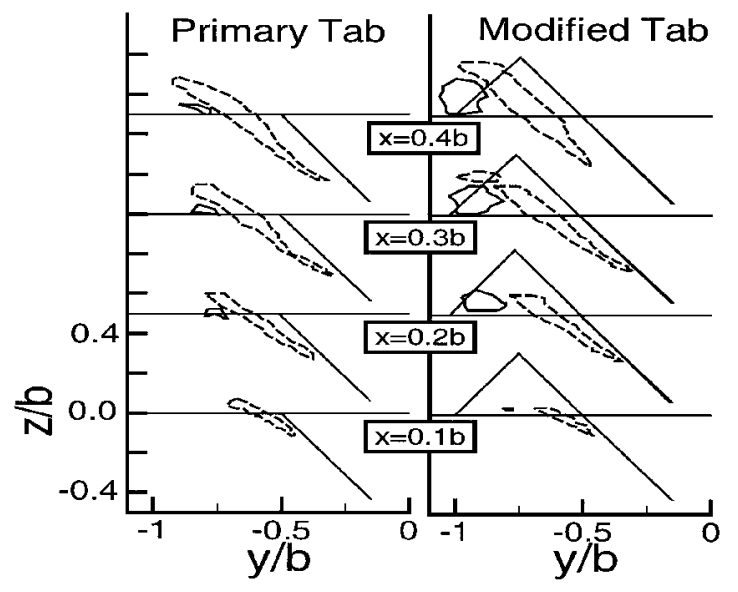

Fig. 8 Cross-vane, streamwise vorticity probe observations; dashed contour bounds the nominal region for $\overline{\omega_{x}}<0$.

permits these magnitudes to be compared between the primary and $\mathrm{P} \& \mathrm{~S}$ tab cases. These data are presented in Fig. 7. It is apparent that: 1) the physical extent of the region in which vorticity is introduced into the flow is unchanged by the secondary tabs and 2) the magnitude of the flux term is significantly enhanced near the junction of the primary and secondary tabs.

\section{Downstream Results}

Qualitative streamwise vorticity. The results of the cross-vane probe survey are presented in Fig. 8. These data show the region of $\omega_{x}>0$ that can be principally ascribed to the reorientation of the approach boundary-layervorticity and the region of $\omega_{x}<0$ that primarily results from the tab-induced pressure hill. A comparison of the primary and the $P \& S$ tab configurations shows that the $\omega_{x}>0$ region is considerablystrengthenedby the presence of the secondary 
tab. This observation is compatible with the modification of the surface pressure field noted earlier. It is pertinent to note that the large pitch and yaw angles of this very near-field region $(0 \leq x / b \leq 0.4)$ precluded quantitative hot-wire measurements. Hence, these qualitative observations are considered to make a valuable contribution to the interpretation of this flowfield.

\section{Quantitative Streamwise Vorticity Results}

The results of the spatially averaged streamwise vorticity calculations [see Eq. (5)] using the $\bar{v}(y, z)$ and $\bar{w}(y, z)$ data at $x / b=1.2$ are presented in Fig. 9. The two regions of vorticity that were shown by the cross-vane probe at the farther upstream stations are also shown in these observations. A comparison of the data from the two geometries indicates that the peak magnitude of the streamwise vorticity from the pressure hill, i.e., $\bar{\omega}_{x}^{*}<0$ region, is slightly higher for the $\mathrm{P} \& \mathrm{~S}$ tab geometry. Specifically, the peak values are ${\overline{\omega_{x}}}^{*}=-1.74$ for the $\mathrm{P} \& \mathrm{~S}$ case and -1.59 for the primary-only case. The ${\overline{\omega_{x}}}^{*}<0$ region is also characterized by a slightly larger area for the $P \& S$ tab case. This implies that there is a somewhat higher total circulation in this region for the $P \& S$ tab geometry. The comparison of the regions that contain the reoriented boundarylayer vorticity in addition to that from the $\mathrm{P} \& \mathrm{~S}$ pressure field of the secondary tab, i.e., the $\overline{\omega_{x}} *>0$ regions, shows that the peak value is significantly higher $\left(\overline{\omega_{x}} *=1.67\right.$ compared to ${\overline{\omega_{x}}}^{*}=1.22)$ and that the area of the region is larger for the $\mathrm{P} \& \mathrm{~S}$ tab geometry. That is, the circulation in this ${\overline{\omega_{x}}}^{*}>0$ region is significantly larger for the $\mathrm{P} \& \mathrm{~S}$ tab geometry. This result shows that the ad-

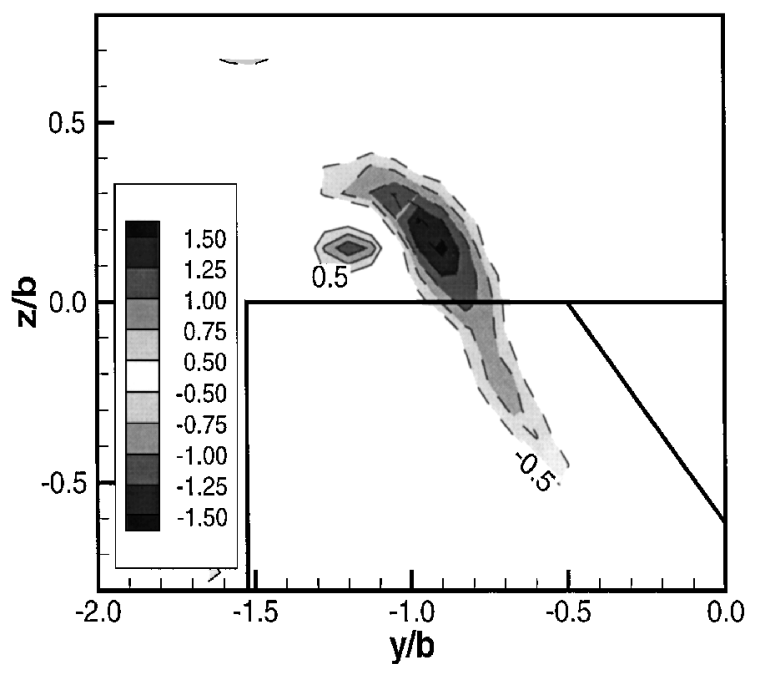

a) Primary tab

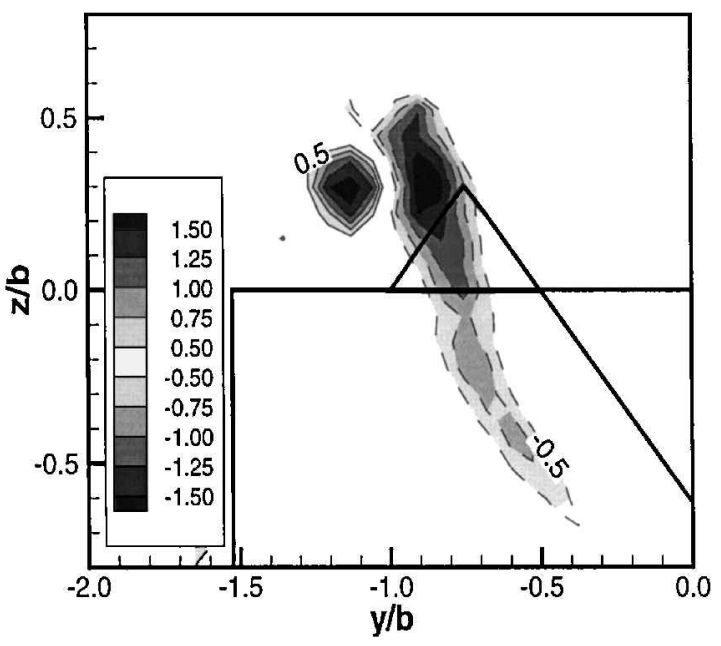

b) $P \& S$ configuration

Fig. 9 Contours of $\bar{\omega}_{x}$ at $x / b=1.2$.

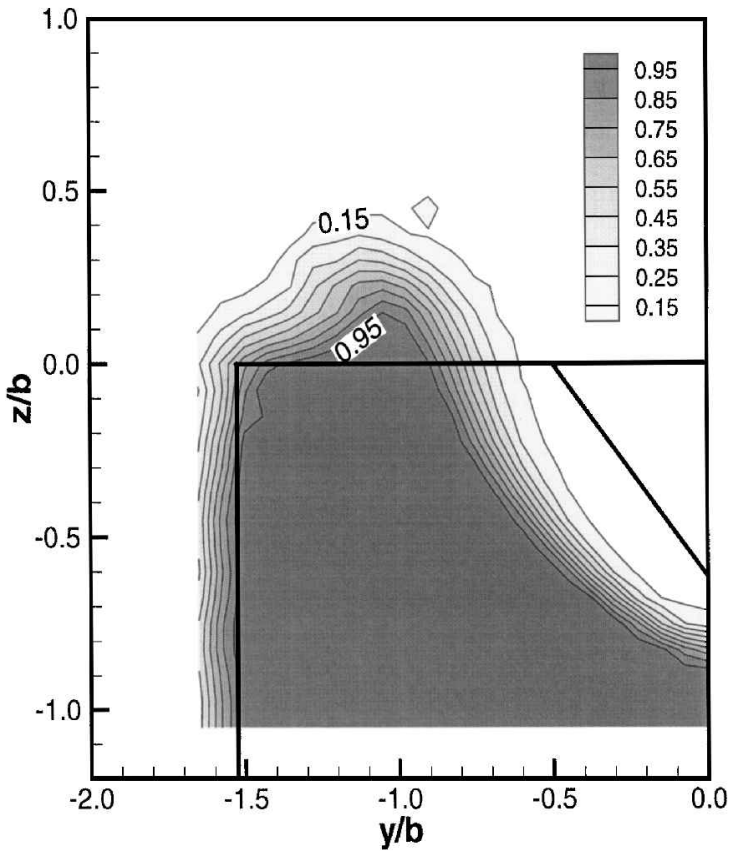

a) Primary tab

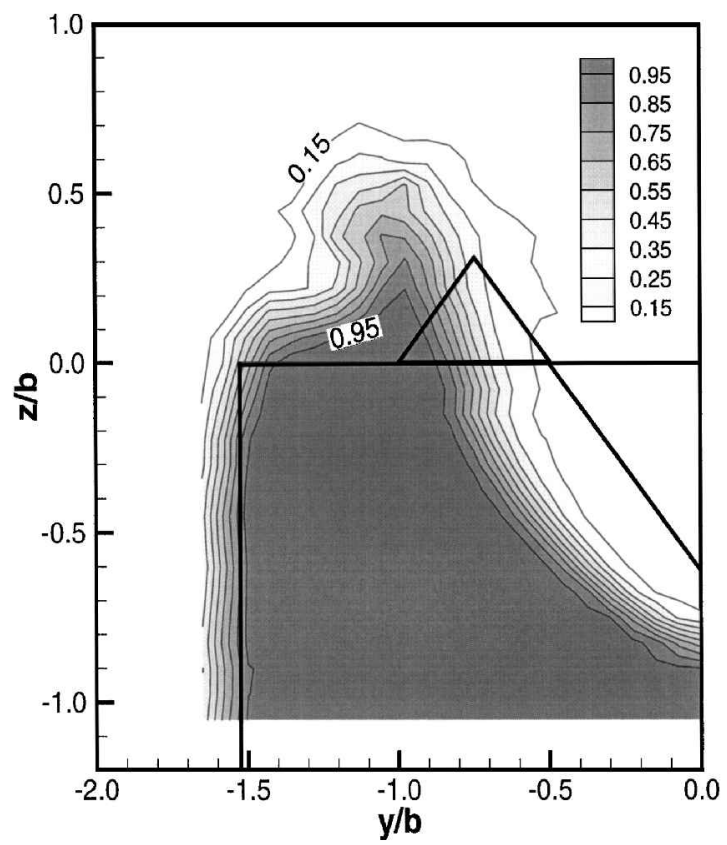

b) $P \& S$ configuration

Fig. 10 Isotachs of the streamwise velocity; $\bar{u}(y, z) / U_{\mathrm{up}}$ at $x / b=1.2$.

ditional vorticity that is introduced into the flow as a result for the secondary tabs is not lost by diffusion into the region of opposite sign vorticity, rather it combines with the streamwise vorticity of the same sign that derives from the reoriented boundary layer.

\section{Streamwise Velocity: Time-Averaged Values at $x / b=1.2$}

The average streamwise velocity contours are shown in Fig. 10; see Ref. 10 for the presentation and discussion of the available results at $x / b=0.74$ (single wire) and 2.0. These data show that the distortion of the shear layer for the primary tab geometry is enhanced for the P\&S tab geometry. As before, the shear layer is aligned with the tunnel side wall. It is now also aligned with the tab projection. By comparing Fig. 10a (primary) and 10b (P\&S), it is apparent that the intended benefit of the secondary tab has been achieved. 


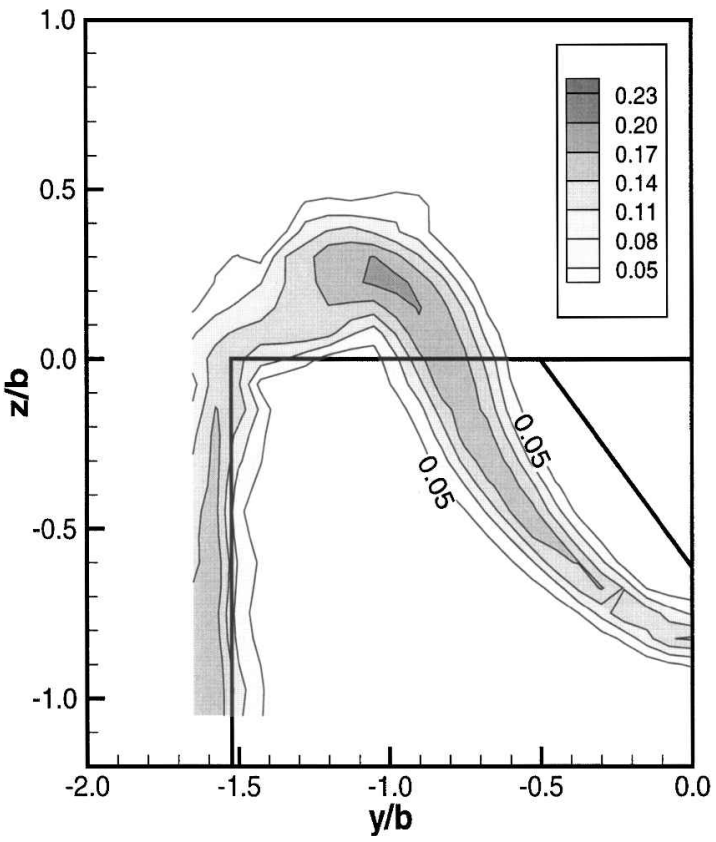

a) Primary tab

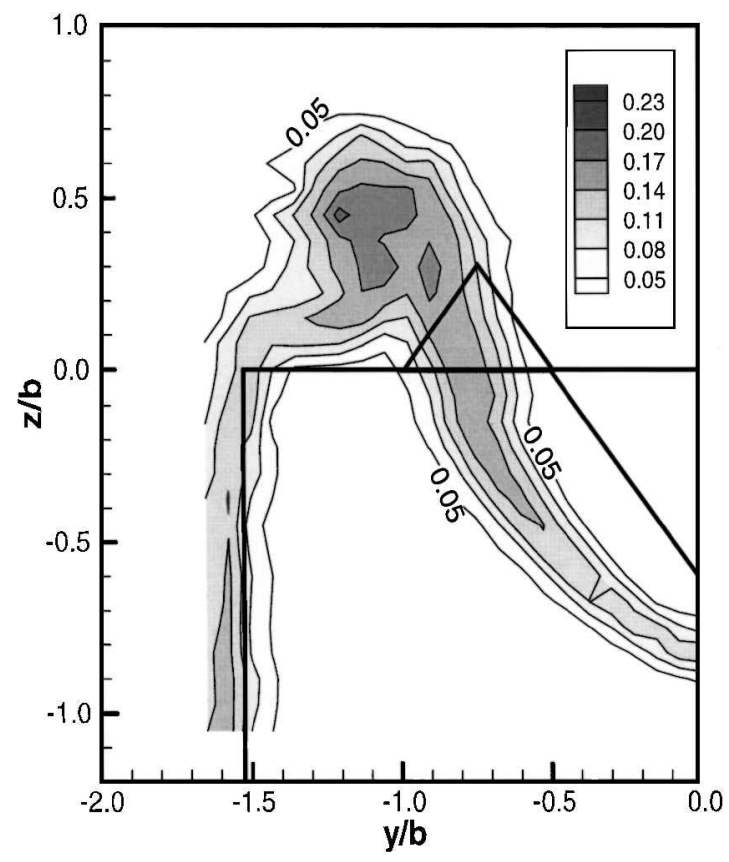

b) $P \& S$ configuration

Fig. 11 Streamwise velocity, fluctuation intensity contours at $x / b=1.2$.

Specifically, high-speed core fluid has been displaced farther into the ambient region as a result of the attachment surface provided by the secondary tab.

\section{Streamwise Velocity: Fluctuation Intensities}

The relative streamwise fluctuation intensity values, i.e., rms levels, designated by the tilde, at $x / b=1.2$ for the primary tab as well as the $P \& S$ configuration are shown in Fig. 11 . The peak intensity value for the untabbed case (not shown here) and for the primary tab case is $0.18=\tilde{u} / U_{\text {up }}$. These values, as noted earlier, are as expected for a single-stream shear layer. This observation suggests that the shear layer of the primary tab can be described as being transported by the induced outflow associated with the tab. In contrast, the peak intensity value of the $\mathrm{P} \& \mathrm{~S}$ configuration is $0.21=\tilde{u} / U_{\text {up }}$. This suggests an active enhancement of the turbulence field in the $P \& S$ case. It is also apparent in these figures that a larger spatial domain of the high fluctuation levels can be attributed to the secondary tabs.

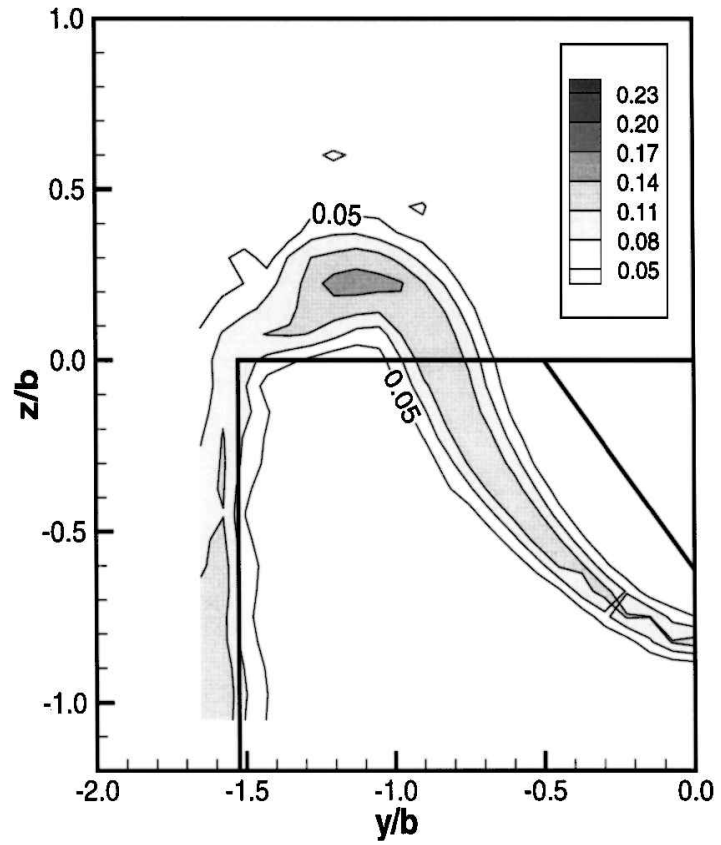

a) Primary tab

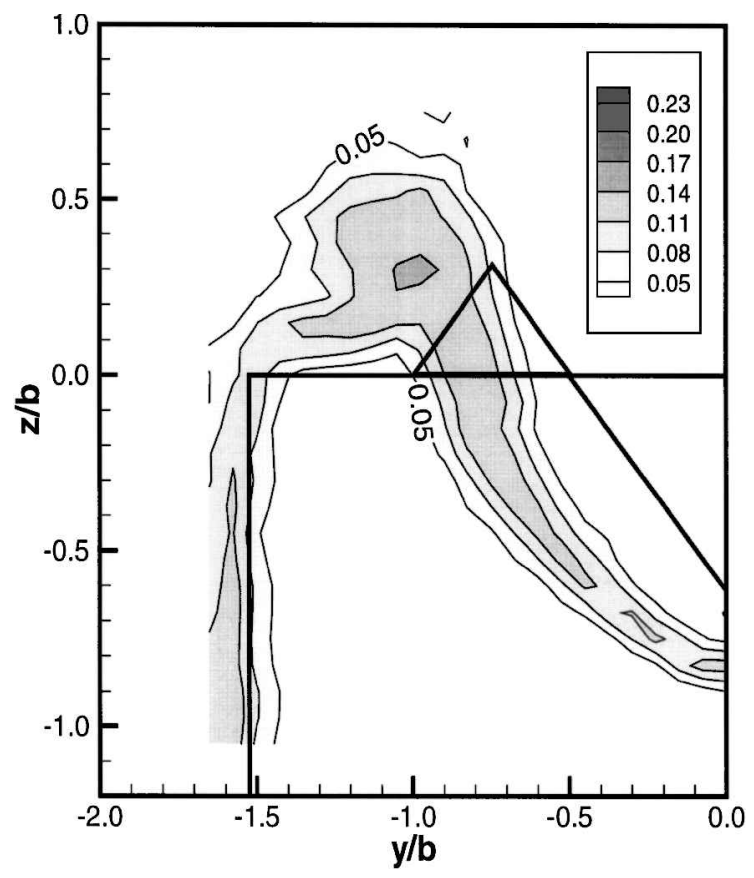

b) $P \& S$ configuration

Fig. 12 Contours of the lateral velocity fluctuations; $\tilde{v} / U_{\text {up }}$ at $x / b=1.2$.

Secondary Flow Velocities: Fluctuation Intensities

Velocity components in the $y$ and $z$ directions will be referred to as secondary flow velocity components. The fluctuation intensity distributions, presented as contour plots, are shown in Fig. 12 for the lateral $(y)$ and in Fig. 13 for the normal $(z)$ directions. These magnitudes are, as expected, smaller than those for the streamwise direction. It is instructive that the peak $\tilde{v} / U_{\text {up }}$ value for the primary tab case $(0.154$ of Fig. 12$)$ is greater than the corresponding $\tilde{w} / U_{\text {up }}$ value (0.141 of Fig. 13), whereas this relationship is reversed for the P\&S configuration: $\tilde{w} / U_{\text {up }}=0.161$ (Fig. 13) and $\tilde{v} / U_{\text {up }}=0.145$ (Fig. 12).

\section{Kinematic Reynolds Shear Stresses}

The $x$-array data, represented in the secondary flow results, also permits the evaluation of the correlations $\overline{u^{\prime} v^{\prime}}$ and $\overline{u^{\prime} w^{\prime}}$. These values, which represent the negative of the kinematic Reynolds shear 


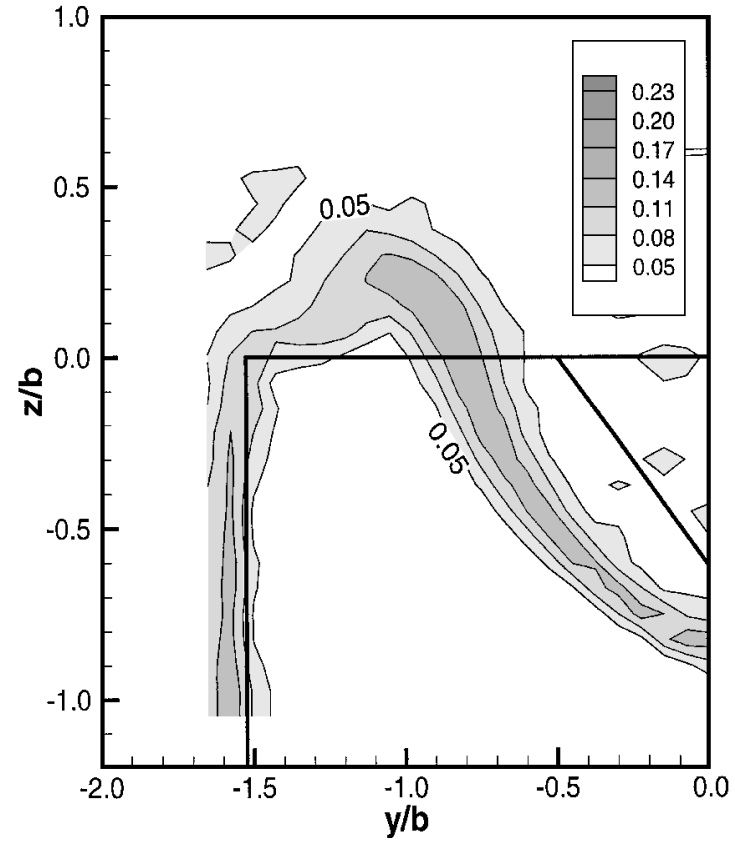

a) Primary tab

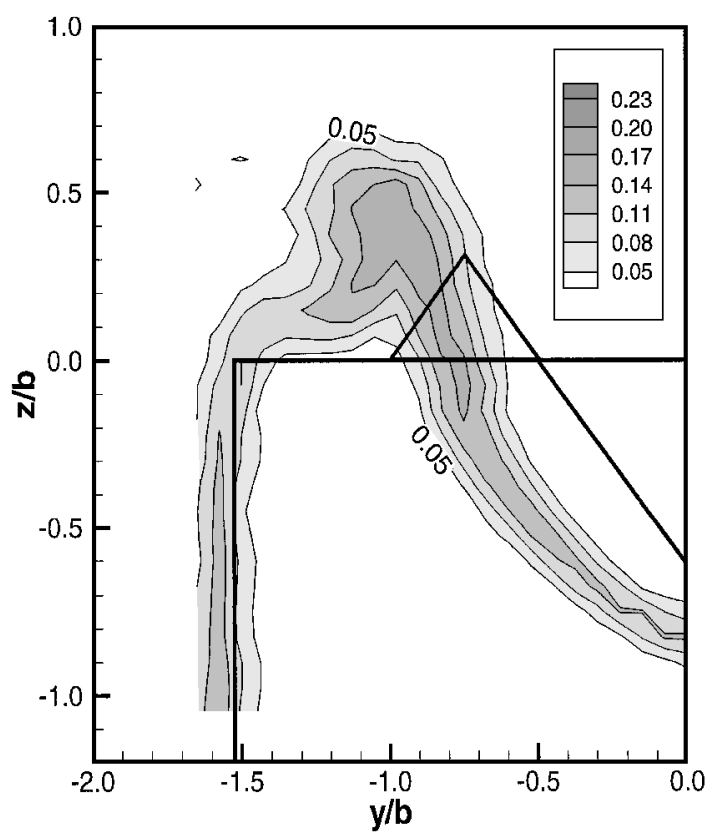

b) $P \& S$ configuration

Fig. 13 Contours of the normal velocity fluctuations; $\tilde{w} / U_{\mathrm{up}}$ at $x / b=1.2$.

stresses, are presented in Figs. 14 and 15. The distribution of $\overline{u^{\prime} v^{\prime}}$ is particularly instructive in showing the respective influence of the two tab configurations. The primary tab $\overline{u^{\prime} v^{\prime}}$ shows negligible shear stress in the shear layer that is lifted from the separation lip by the combined $\overline{\omega_{x}}>0$ and $\overline{\omega_{x}}<0$ vortex motions. In contrast, the P\&S configuration shows the distinct presence of a peak in the $\bar{u}(y)$ distribution that is associated with the corresponding shear stress and the production of $\overline{q^{2}}$.

\section{Turbulence Kinetic Energy}

The fluctuation intensity results can be combined to represent the turbulence kinetic energy $\overline{q^{2}} / 2$. Specifically,

$$
\frac{\overline{q^{2}}}{2}=\left[\frac{\tilde{u}^{2}+\tilde{v}^{2}+\tilde{w}^{2}}{2}\right]
$$

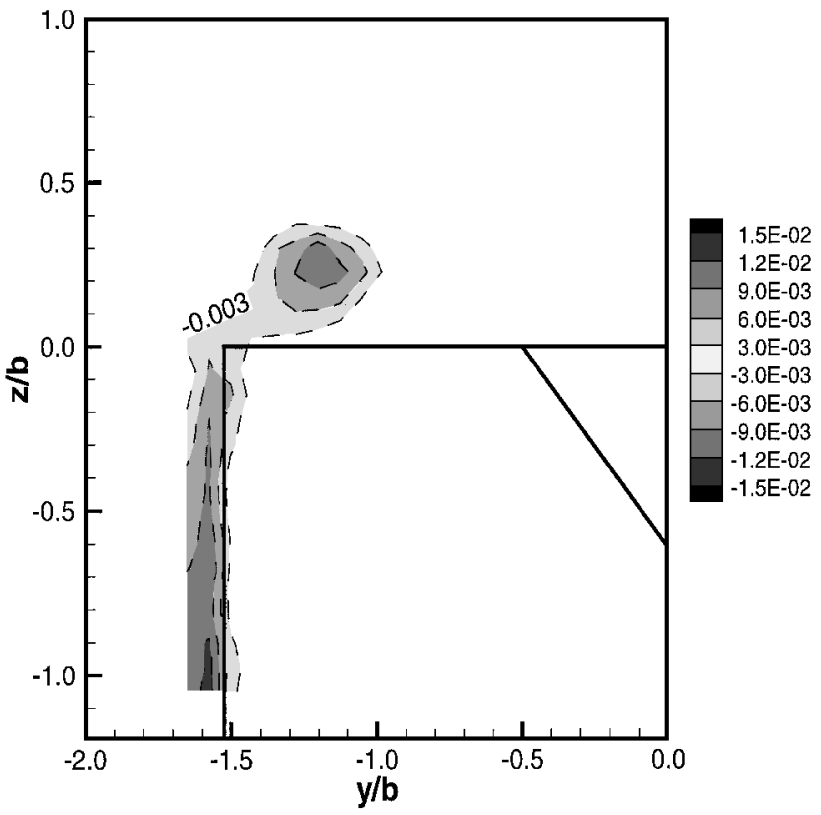

a) Primary tab

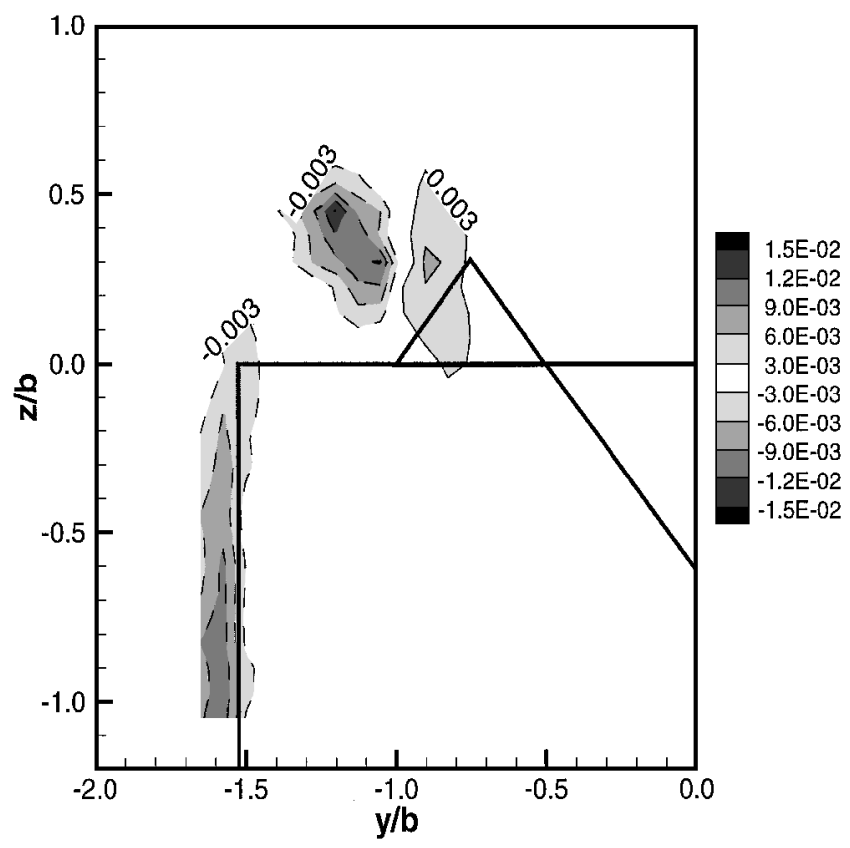

b) $P \& S$ configuration

Fig. 14 Negative kinematic $x-y$ Reynolds stress; $\overline{u^{\prime} v^{\prime}} / U_{\text {up }}$ at $x / b=1.2$.

These data are presented in Fig. 16; they also show that $\overline{q^{2}}$ is larger in magnitude and greater in extent for the $P \& S$ tab case.

\section{Secondary Flow Velocities: Time-Average Values}

The $\bar{v}$ and $\bar{w}$ distributions have been combined to represent the projected, onto the $y-z$ plane, time-average velocity vectors; these distributions are shown in the scaled portions of Fig. 17. Note, the measured data $(y \leq 0)$ have been represented as a symmetrical distribution for the unscaled $(y \geq 0)$ representations. The laterally redirected core flow is quite apparent in these distributions, and its strengthened character for the $\mathrm{P} \& \mathrm{~S}$ configuration is also apparent. The influence of the secondary tab on the normal $\bar{w}$ component values is strongly evident in Fig. 17b.

These data are also used to identify the streamline patterns; the directional characteristics of the pattern have been emphasized by using uniform vector lengths in this second representation. This alternative representation is presented in the unscaled portions of Fig. 17. (Note the coordinate system is rotated for Fig. 17.) The 


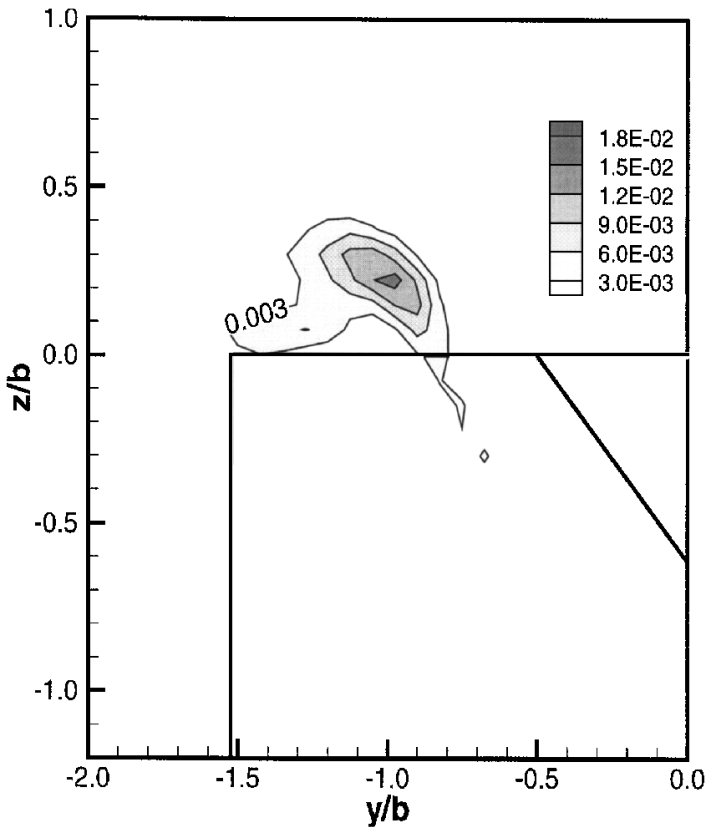

a) Primary tab

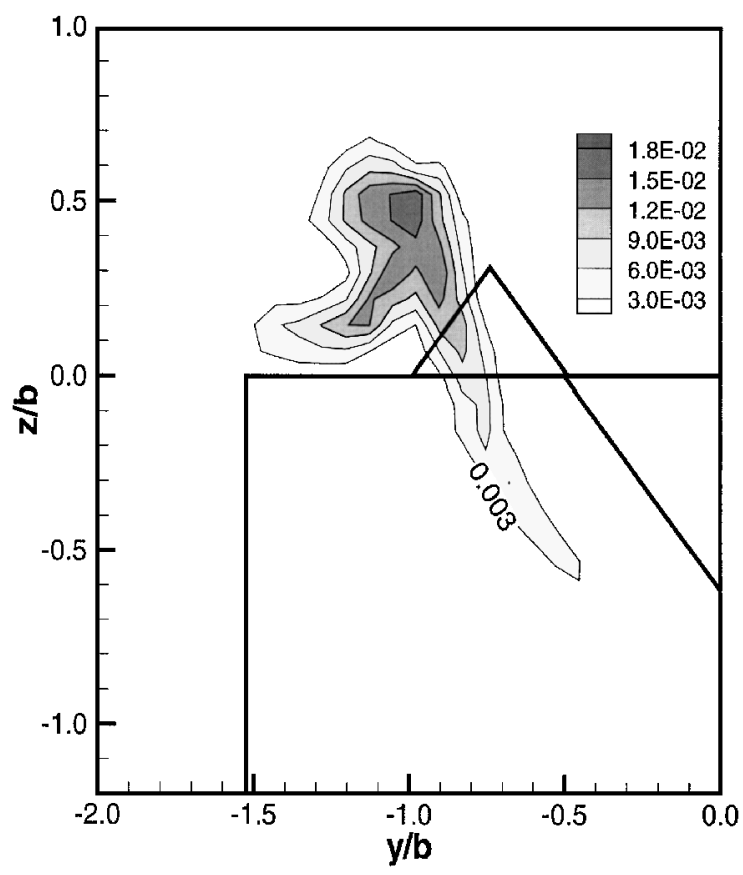

b) $P \& S$ configuration

Fig. 15 Negative kinematic Reynolds stress; $\overline{u^{\prime} w^{\prime}} / U_{\text {up }}$ at $x / b=1.2$.

presence of a large-scale vortex motion, centered at $y / b=-0.85$ and $z / b=0.43$, is quite evident in Fig. 17.

\section{Length Scales of the Streamwise Velocity Field}

Two length parameters have been established to aid in the interpretation of the streamwise velocity contour data. The first of these measures, $\delta_{\text {ambient }}$, is defined as the maximum $z$ location of the $0.15 U_{\text {up }}$ contour. The second of these measures, $\delta_{\text {core }}$, is defined by the $z$ location of the $0.95 U_{\text {up }}$ contour for $y=0$, i.e., along the centerline of the tab. Graphical representations of the quantities are provided by Bohl and Foss ${ }^{10}$; space limitations preclude their inclusion herein. These data show that the penetration of the shear layer into the ambient region with the P\&S tab geometry is nominally $500 \%$ higher than with the untabbed geometry and 166\% higher than that of the primary tab geometry at $x=0.7 b$ (the most upstream measurement location). These data also show that the depth of penetration of the shear layer into the core region is essentially

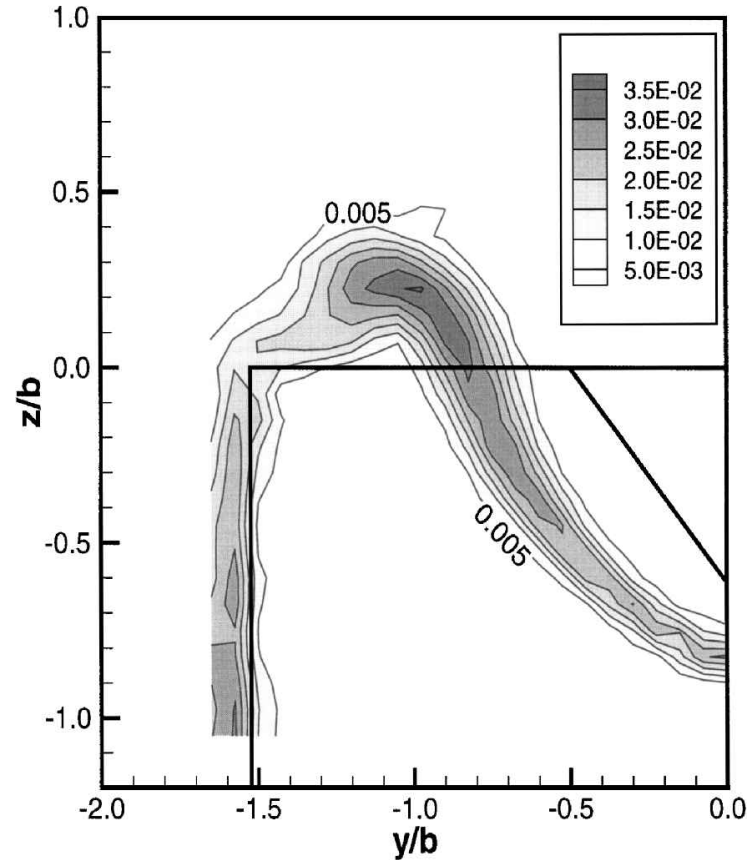

a) Primary tab

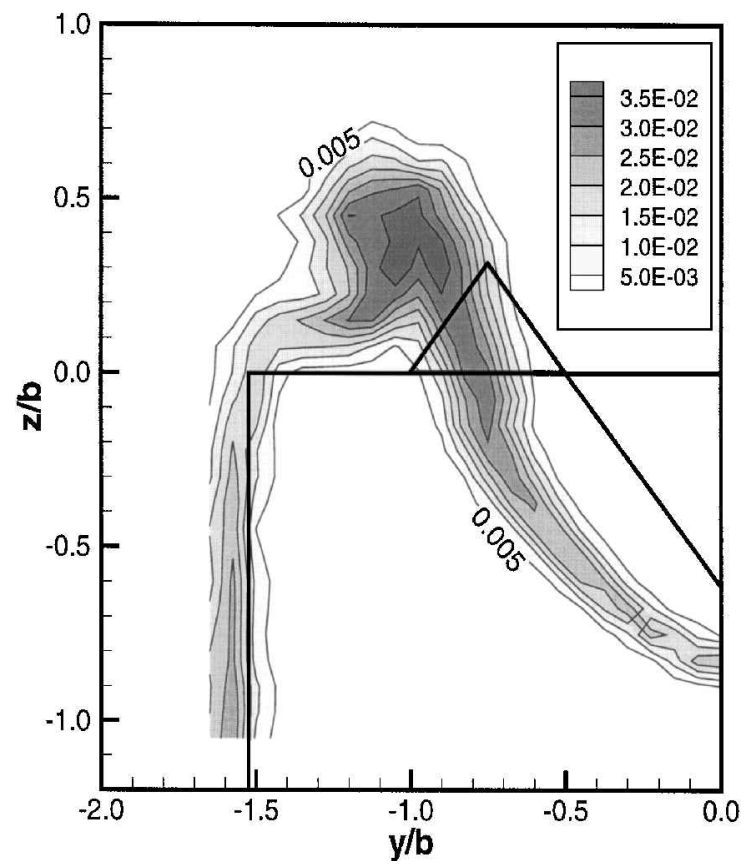

b) $P \& S$ configuration

Fig. 16 Turbulence kinetic energy; $\left(\tilde{u}^{2}+\tilde{v}^{2}+\tilde{w}^{2}\right) / 2$ at $x / b=1.2$.

the same for both the primary tab geometry and the P\&S. As shown by Bohl and Foss, ${ }^{10}$ the shear-layerpenetration (as given by $\delta_{\text {core }}$ ) is nominally $600 \%$ greater for both tabbed geometries when compared to the untabbed geometry at $x=0.7 b$.

The shear-layer momentum thickness was also calculated for the investigated geometries. The shear-layer momentum thickness $\theta$ is defined as

$$
\theta(x, y)=\int u^{*}\left(1-u^{*}\right) \mathrm{d} s^{\prime}
$$

where $u^{*}$ is defined as $\left(\bar{u}-u_{\min }\right) /\left(u_{\text {up }}-u_{\min }\right), u_{\min }$ was taken to be $1.5 \mathrm{~m} / \mathrm{s}$, and $s^{\prime}$ is a distance between the high-speed $\left(u_{\text {up }}\right)$ and low-speed $\left(u_{\text {min }}\right)$ limits of the integration along a line of constant $x$ and $y$. This definition of $u^{*}$ was adopted to address the low velocities encountered at the low-speed edge of the shear layer. The shear-layer momentum thickness was calculated in two regions of the flowfield: 1) in the shear layer below the projected tip of the tab, 


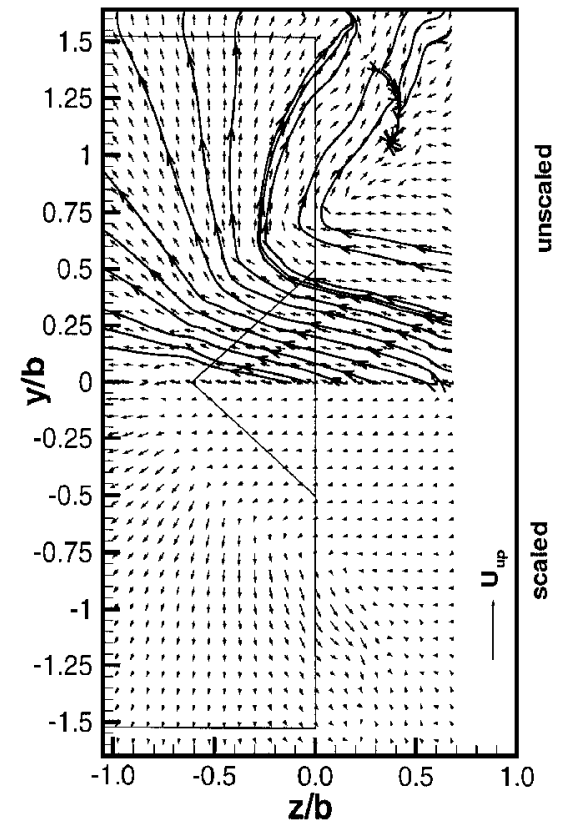

a) Primary tab

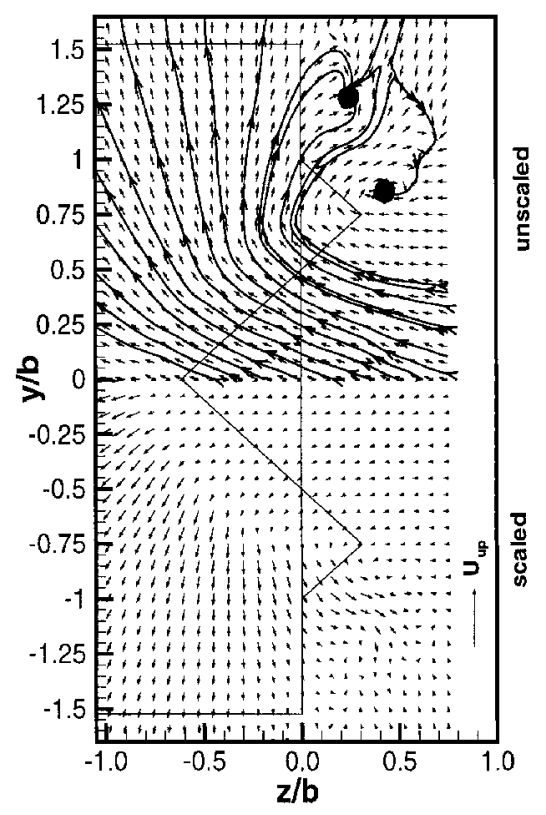

b) $\mathrm{P} \& \mathrm{~S}$ configuration

Fig. 17 Secondary flow vectors.

i.e., for $y=0$, and 2) through the plume. In the plume region, the $y$ location of the traverse was selected to allow a $z$ direction traverse to be nominally perpendicular to the isotachs.

The results for the plume region are shown in Fig. 18. The plume region is characterized by the primary vertical $(z>0)$ distortion of the isotachs as shown in Fig. 10. These data indicate significant differences in the flowfields for the no-tab, primary tab, and $\mathrm{P} \& S$ tab geometries. Specifically, the magnitude of the momentum thickness is the greatest for the P\&S tab geometry and least for the untabbed geometry. It is instructive that the growth rate was found to be $\mathrm{d} \theta / \mathrm{d} x=0.050$ for the $P \& S$ geometry and $\mathrm{d} \theta / \mathrm{d} x=0.035$ for both the untabbed geometry and primary tab geometry. That is, the latter two cases exhibit $\mathrm{d} \theta / \mathrm{d} x$ values equivalent to the values observed by Liepmann and Laufer ${ }^{16}$ and Bruns et al. ${ }^{15}$ for conventional single-stream shear layers.

The shear-layer momentum thickness in the tip region is smaller than that for the untabbed geometry at the same location. This observation is rational given that the shear layer starts where the physical surface, i.e., the tab, ends (nominally $x=0.61 b$ ) and, therefore, the tabbed shear layer (at $y=0$ ) has a shorter fetch for develop-

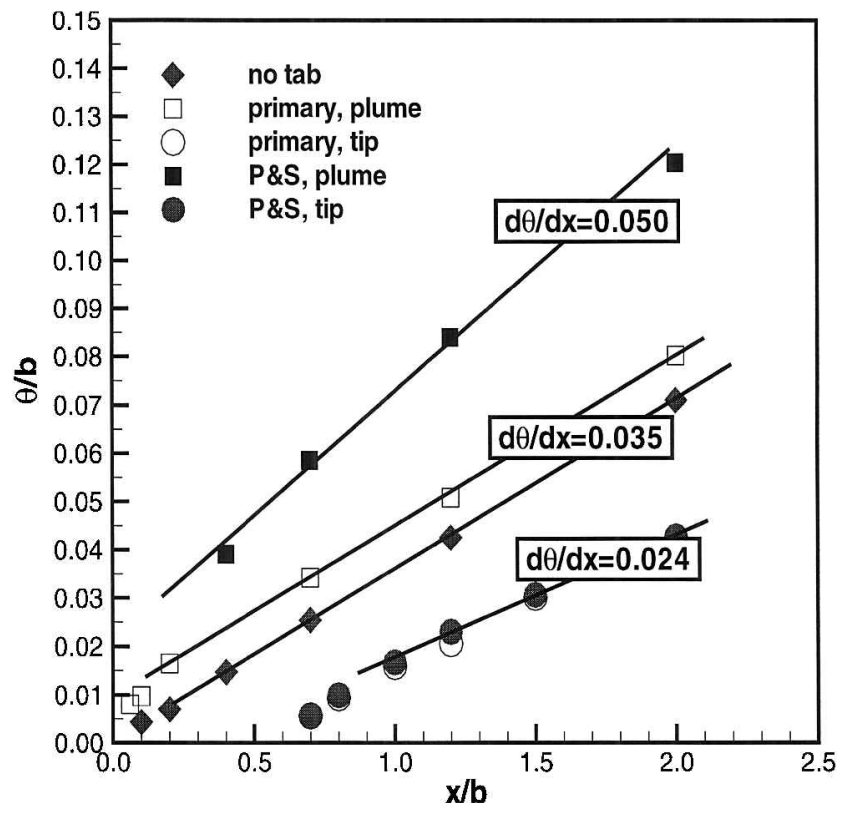

Fig. 18 Momentum thickness distributions; plume survey at $y / b=-1$, tip survey at $y / b=0$.

ment. The growth rate of the shear layer at $y=0$ was found to be $\mathrm{d} \theta / \mathrm{d} x=0.024$. The lower growth rate (with respect to that of the untabbedjet) can be attributed to the growth of the shear layer, which is principally into the high-momentum core flow. It is instructive to note that the shear layer thickness and growth rates at $y=0$ are identical for the primary tab and $P \& S$ tab geometries.

\section{Summary and Conclusions}

Distortions in the isotachs, and the presence of concentrated regions of streamwise vorticity that result from the introduction of one or more tabs at the exit plane of freejets, have been well established in previous investigations. The isolated, i.e., a single tab, and the large-scale tab in the present investigation have permitted a more detailed documentation of the near field, $|x| b \mid<2$, than was feasible in these prior studies. The present study has also documented the significant enhancement of these near-field attributes when secondary tabs are placed adjacent to (and on both sides of) the primary tab. Specifically, the pressure, velocity, and vorticity fields have been documented for equilateral triangular secondary tabs, with a base length that is $50 \%$ of the base length of the primary tab. The secondary tabs were directed away from the primary tab at an (optimized) angle of $40 \mathrm{deg}$ with respect to the streamwise direction.

An examination of the growth rate $\mathrm{d} \theta / \mathrm{d} x$ and the turbulence field properties for the $\mathrm{P} \& \mathrm{~S}$ cf the primary tab configurations suggests that the secondary tabs significantly modify the separating shear layer for the former. In contrast, the shear layer of the primary tab appears to be lifted upward (normal to) the plane of the jet nozzle.

The inner concentrated region of $\overline{\omega_{x}}$ can be traced to the pressure field upstream of the tab; the outer region derives from the reorientation of the approach boundary layer and was reinforced by the pressure minimum near the exit plane and (nominally) centered at the secondary tab.

The use of secondary tabs will enhance the contribution of a given primary tab and, if desired, a smaller primary tab could be used for the same beneficial effects. For example, a smaller thrust loss for a given level of mixing in a given nozzle system could be obtained.

\section{Acknowledgments}

The support of the NASA Lewis Research Center, Grant NAG 3-1459, and the beneficial technical consultations with the Grant Monitor, Khairul B. Zaman, are gratefully acknowledged by the authors. 


\section{References}

${ }^{1}$ Bradbury, L. J. S., and Khadem, A. H., "The Distortion of a Jet by Tabs," Journal of Fluid Mechanics, Vol. 70, 1975, pp. 801-813.

${ }^{2}$ Ahuja, K. K., and Brown, W. H., "Shear Flow Control by Mechanical Tabs," AIAA Paper 89-0994, 1989.

${ }^{3}$ Zaman, K. B. M. Q., Reeder, M. F., and Samimy, M., "Control of an Axisymmetric Jet Using Vortex Generators," Physics of Fluids, Vol. 6, June 1994, pp. 778-793.

${ }^{4}$ Reeder, M. F., and Samimy, M., "The Evolution of a Jet with VortexGenerating Tabs: Real-Time Visualization and Quantitative Measurements," Journal of Fluid Mechanics, Vol. 311, 1996, pp. 73-118.

${ }^{5}$ Lighthill, M. J., "Introduction to Boundary Layer Theory," Laminar Boundary Layers, edited by L. Rosenhead, Clarendon, Oxford, England, UK, 1963, Chap. 2.

${ }^{6}$ Panton, R. L., Incompressible Flow, Wiley, New York, 1984.

${ }^{7}$ Bohl, D. G., and Foss, J. F., "Characteristics of the Velocity and Streamwise Vorticity Fields in a Developing Tabbed Jet," AIAA Paper 95-0102, Jan. 1995.

${ }^{8}$ Bohl, D. G., and Foss, J. F., "Enhancement of Passive Mixing Tabs by the Addition of Secondary Tabs," AIAA Paper 96-0545, Jan. 1996.

${ }^{9}$ Yu, S. C. M., and Hou, Y. X., "Near-Field Velocity Measurements of Confined Square Jet with Primary and Secondary Tabs," AIAA Journal, Vol. 36, No. 2, 1998, pp. 288-289.
${ }^{10}$ Bohl, D. G., and Foss, J. F., "An Experimental Study of the Near Field Region of a Free Jet with Passive Mixing Tabs," NASA CR-97-206296, Nov. 1997.

${ }^{11}$ Collis, D. C., and Williams, M. J., "Two-Dimensional Convection from Heated Wires at Low Reynolds Number," Journal of Fluid Mechanics, Vol. 6, 1959, pp. 357-384.

${ }^{12}$ Foss, J., Wallace, J., and Wark, C., "Vorticity Measurements," Handbook of Fluid Dynamics and Fluid Machinery, edited by J. A. Schetz and A. E. Fuhs, Vol. 2, Wiley, New York, 1996, pp. 1064-1078.

${ }^{13}$ Wygnanski, I., and Fielder, H. E., "The Two-Dimensional Mixing Region," Journal of Fluid Mechanics, Vol. 41, Pt. 2, 1970, pp. 327-361.

${ }^{14}$ Hussain, F., and Zaman, K., "An Experimental Study of Organized Motions in the Turbulent Mixing Layer," Journal of Fluid Mechanics, Vol. 159, 1985, pp. 85-104.

${ }^{15}$ Bruns, J. M., Haw, R. C., and Foss, J. F., "The Velocity and Transverse Vorticity Field in a Single Stream Shear Layer," Eighth Symposium on Turbulent Shear Flows (Munich, Germany), Turbulent Shear Flow Symposium, Paper 3-1, 1991.

${ }^{16}$ Liepmann, H., and Laufer, J., "Investigation of Free Turbulent Mixing," NACA Rept. 1257, Aug. 1947.

F. W. Chambers Associate Editor 